\title{
CARACTÉRISATION MINÉRALOGIQUE ET CHIMIQUE DE VITRIFIATS DE REFIOM IMPORTANCE DE LA LOCALISATION DES ÉLÉMENTS POLLUANTS
}

\author{
Cécile Mahé-Le Carlier, Alain Ploquin, Jean-Jacques Royer
}

Centre de recherches pétrographiques et géochimiques, CNRS Nancy

La loi du 13 juillet 1992 prévoyant qu'à la date du I ${ }^{\text {er }}$ juillet 2002, seul les résidus ultimes seront autorisés en décharge de classe I, a conduit au développement de travaux concernant leur stabilisation. Deux types de vitrifiats de Refiom ont été élaborés avec un chimisme et une minéralogie différents. L'étude a montré l'importance de certains cristaux stabilisant certains éléments polluants tandis que d'autres phases rendent le matériau peu stable.

According to the law of July 15, 1992, only ultimate wastes can be accepted in class I rubbish dumps after July I, 2002. This has led to studies concerning the stabilization of such wastes. Two types of vitrified waste (vitrifiats de REFIOM) have been studied, each with a different chemistry and mineralogy. This work shows the importance of some crystals that allow a good stabilization of certain polluant elements whereas other phases can lead to an unstable material.

\section{INTRODUCTION}

La protection de l'environnement et la limitation de la pollution induite par l'activité humaine sont des préoccupations majeures de notre décennie. La loi du 13 juillet 1992 prévoit qu'à la date du ${ }^{\text {er }}$ juillet 2002, seules les décharges de classe I seront autorisées en France à accueillir les résidus ultimes. Pout y être admis, ils doivent répondre au test de lixiviation (norme $X 3|-2| 0$ ). Les résidus d'épuration des fumées d'ordures ménagères (Refiom) ne répondent pas à ce test et doivent donc être stabilisés. Actuellement, une partie de ces cendres y est admis après avoir été incorporée dans des matrices cimentaires. Un autre moyen de stabilisation est la transformation en vitrifiats qui pourraient éventuellement être valorisés, en sous-couches routières notamment. Le retraitement de ces Refiom est un problème important quand on considère que $400000 \mathrm{t}$ de résidus sont produites annuellement en France ${ }^{[l]}$.

\section{MATERIEL ET METHODE}

\section{Matériel}

Un procédé de traitement thermique des Refiom par arc électrique a été développé au centre de recherche d'EDF des Renardières. Ce procédé consiste à porter les Refiom à une température de $1450^{\circ} \mathrm{C}$ permettant leur fusion ${ }^{[2]}$.

Deux types de vitrifiats ont été étudiés : des échantillons essentiellement vitreux et d'autres cristallisée ${ }^{[3]}$. La différence entre les deux produits vient de la nature des produits ajoutés aux cendres. L'ensemble des échantillons a été élaboré à partir de Refiom dans les mêmes conditions à $1450{ }^{\circ} \mathrm{C}$. La texture vitreuse ou cristallisée provient principalement de la différence de composition chimique des produits de départ. Le refroidissement s'est effectué dans des creusets en fonte, sans trempe. Macroscopiquement, les vitrifiats à dominante vitreuse sont homogènes, de couleur noire. Les vitrifiats « cristallisés » présentent une bordure vitrifiée sur 2-3 $\mathrm{mm}$ : la texture du matériau restant est homogène, pierreux et de couleur grise ; de grosses bulles aplaties $(0,5$ à $2 \mathrm{~cm})$ sont présentes à $4-5 \mathrm{~cm}$ en dessous de la surface de la coulée.

\section{Chimie}

Dans un premier temps, les échantillons sont broyés à moins de $70 \mu \mathrm{m}$, puis $100 \mathrm{mg}$ de poudre d'échantillon sont fondus dans un creuset en platine avec $900 \mathrm{mg}$ de métaborate de lithium et $600 \mathrm{mg}$ d'acide borique. Le mélange obtenu est ensuite dissout dans de l'acide nitrique. Les éléments majeurs ( $\mathrm{Si}, \mathrm{Al}, \mathrm{Fe}, \mathrm{Mn}, \mathrm{Mg}, \mathrm{Ca}, \mathrm{Na}, \mathrm{K}, \mathrm{Ti}$, et $\mathrm{P}$ ) sont analysés par spectrométrie d'émission atomique couplée à une induction plasma (ICP-AES), tandis que les éléments en traces sont obtenus par spectrométrie de masse couplée à une induction plasma (ICP-MS). Les tests de lixiviation sont réalisés dans des béchers en polypropylène à température ambiante. Les monolithes, taillés dans les vitrifiats bruts, sont polis et plongés dans une solution d' $\mathrm{HCl}$ à $\mathrm{pH} \mathrm{I}$. Après expérience, les solutions sont filtrées à $0,45 \mu \mathrm{m}$ puis acidifiées avant analyse. Les éléments en solution sont dosés comme précédemment. 


\section{Minéralogie}

L'observation microscopique nécessite des lames polies d'épaisseur $30 \mu \mathrm{m}$. Celles-ci ont également servi pour les micro-analyses à la microsonde électronique (Cameca SX50, Faculté des sciences de Nancy) équipée de quatre spectromètres dispersifs en longueur d'onde (WDS). Les analyses sont effectuées sous une tension d'accélération de $15 \mathrm{kV}$ et un courant de $10 \mathrm{nA}$. Le calibrage est effectué sur standard géologique.

\section{RÉSULTATS}

\section{Chimie}

Les analyses chimiques globales montrent une très nette différence de composition entre les vitrifiats de Refiom « cristallisés » et « vitreux 》 (tableau I), au niveau des éléments majeurs, notamment pour $\mathrm{SiO}_{2}$ et $\mathrm{CaO}$. Les échantillons « vitreux », homogènes en composition, contiennent également de très fortes teneurs en éléments toxiques $(\mathrm{Cr}$ et $\mathrm{Sn}$, aux environs de $3000 \mathrm{ppm}, \mathrm{Pb}$ aux alentours de 5000 ppm, et Zn dépassant les 40000 ppm). La composition chimique des échantillons « cristallisés " présente, quant à elle, une variation aussi bien pour les teneurs des éléments majeurs tels que $\mathrm{Fe}_{2} \mathrm{O}_{3}\left(0,3\right.$ à $\mathrm{I}, 3 \%$ poids) et $\mathrm{P}_{2} \mathrm{O}_{5}(0,7$ à $\mathrm{I}, 5 \%$ poids), que pour plusieurs éléments traces comme $\mathrm{Cr}$ (5000 à 8000 ppm), Cu (63 à 224 ppm), Sb et Sn (de traces à 7 et 32 ppm respectivement) et $\mathrm{Zn}$ (300 à 1600 ppm). II faut

\begin{tabular}{|c|c|c|c|}
\hline \multicolumn{4}{|c|}{$\begin{array}{l}\text { Tableau I : Composition chimique globale } \\
\text { (-) en dessous du seuil de détection }\end{array}$} \\
\hline & Crist. & Crist. & Vit. \\
\hline $\mathrm{SiO}_{2}(\% \mathrm{pds})$ & 30,74 & 31,20 & 40,74 \\
\hline $\mathrm{Al}_{2} \mathrm{O}_{3}$ & 21,16 & 20,88 & 19,92 \\
\hline $\mathrm{Fe}_{2} \mathrm{O}_{3}$ & 1,33 & 0,36 & 4,74 \\
\hline $\mathrm{MnO}$ & 0,09 & 0,09 & 0,09 \\
\hline $\mathrm{MgO}$ & 3,84 & 3,83 & 1,63 \\
\hline $\mathrm{CaO}$ & 36,23 & 37,73 & 24,17 \\
\hline $\mathrm{Na}_{2} \mathrm{O}$ & 1,10 & 1,08 & 1,75 \\
\hline $\mathrm{K}_{2} \mathrm{O}$ & 0,07 & 0,05 & 1,61 \\
\hline $\mathrm{TiO}_{2}$ & 2,74 & 2,53 & 1,14 \\
\hline $\mathrm{P}_{2} \mathrm{O}_{5}$ & 1,48 & 0,71 & 0,69 \\
\hline PF & 0,00 & 0,67 & $-0,03$ \\
\hline Total & 98,78 & 99,13 & 96,45 \\
\hline As (ppm) & 2,66 & - & 42 \\
\hline $\mathrm{Ba}$ & 1772 & 1573 & 1642 \\
\hline $\mathrm{Cd}$ & 2,13 & 1,42 & 20.6 \\
\hline $\mathrm{Ce}$ & 52,23 & 47,6 & 100,5 \\
\hline $\mathrm{Cr}$ & 8349 & 4831 & 3363 \\
\hline $\mathrm{Cu}$ & 224 & 63,1 & 1161 \\
\hline $\mathrm{Ni}$ & 10,3 & 6,69 & 107 \\
\hline $\mathrm{Pb}$ & 0,37 & - & 5165 \\
\hline $\mathrm{Sb}$ & 7,16 & - & 849 \\
\hline Sn & 31,6 & - & 3269 \\
\hline $\mathrm{Zn}$ & 1587 & 320 & 42687 \\
\hline
\end{tabular}

remarquer qu'à une plus faible teneur en $\mathrm{P}$, sont associées des valeurs inférieures au seuil de détection de I'ICP-MS pour As, Sb, Sn et Pb. L'implication de cette observation est développée dans les paragraphes qui suivent. Ces variations semblent dues à une hétérogénéité intrinsèque de ces échantillons partiellement cristallisés. Cette nette différence de composition chimique entre les échantillons vitreux et cristallisés montre que si la technique employée est la même, ces deux types de vitrifiats sont élaborés à partir de matériaux de compositions initiales différentes.

\section{Minéralogie}

Les échantillons "vitreux 》 sont constitués d'une matrice vitreuse (photo I a) dans laquelle se sont formés des microcristaux automorphes (5 à $20 \mu \mathrm{m}$ ) (photo I b), appartenant à la famille des spinelles (gahnite $\mathrm{ZnAlO}_{4}$ - zinco-chromite $\mathrm{ZnCr}_{2} \mathrm{O}_{4}$ ). Le verre est homogène en composition (déterminée au moyen de la microsonde électronique sur les éléments majeurs) et très proche de celle de l'analyse globale du matériau.

Les échantillons « cristallisés » présentent une texture homo-

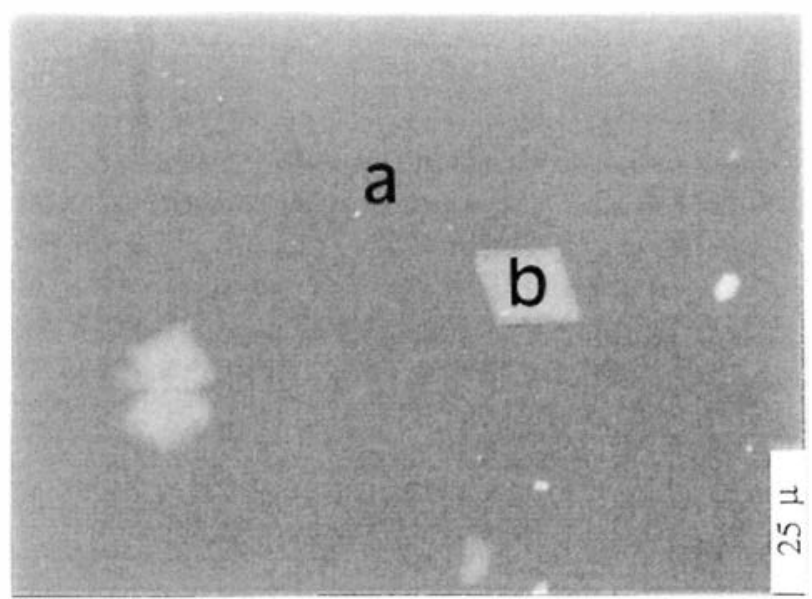

Photo I : Vitrifiat « vitreux » : cristaux de spinelle (b) dans la matrice vitreuse (a)

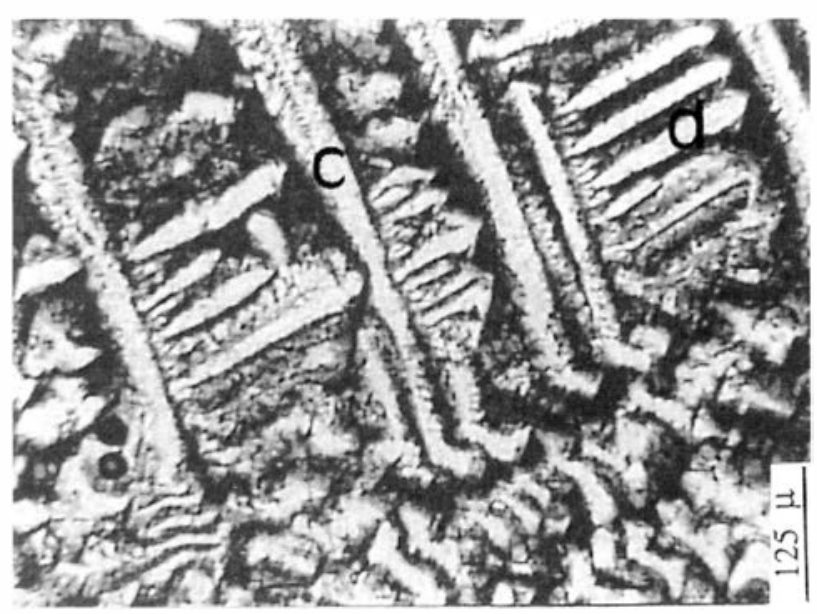

Photo 2 : Vitrifiat « cristallisé » : assemblage de cristaux de mélilites (c) et sphènes (d) dans une matrice vitreuse 


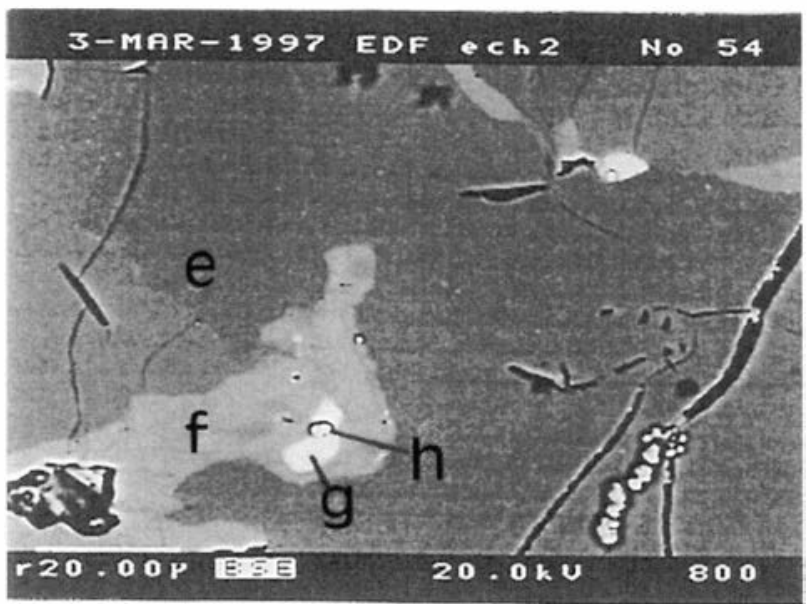

Photo 3 : Vitrifiat « cristallisé » : bille polyphasée : phases majeure (e), interstitielle (f) et arrondies (g), petites phases rondes $(h)$

gène constituée de petits cristaux automorphes de spinelle dispersés dans l'ensemble de l'échantillon et pouvant être inclus dans les phases silicatées. Ils appartiennent à la famille des spinelles (spinelle $\mathrm{MgAl}_{2} \mathrm{O}_{4}$ - magnésiochromite $\mathrm{MgCr}_{2} \mathrm{O}_{4}$ ). La phase cristallisée dominante est un silicate alumino-calcique, la géhlénite (famille des mélilites) se présentant sous forme de grands cristaux à tendance dendritique (photo 2 c). Entre ceux-ci se disposent des cristaux en forme de tablette, appartenant à la famille des sphènes, un silicate titanifère (photo $2 \mathrm{~d}$ ). Après cristallisation, le liquide résiduel s'est solidifié en une matrice vitreuse. Ce verre contient des inclusions arrondies, opaques en lumière transmise. Ce sont des sulfures et des alliages métalliques. Ils ne sont jamais présents dans les cristaux. A proximité des bulles, des billes de diamètre millimétrique (jusqu'à $5 \mathrm{~mm}$ ) à éclat métallique, sont présentes. Elles sont quadriphasées : une phase majeure (photo $3 \mathrm{e}$ ), une phase interstitielle à contours arrondis (photo $3 \mathrm{f}$ ) (tous deux des phosphures) dans laquelle se situe une phase ronde encore plus petite (photo $3 \mathrm{~g}$ ) (alliage métallique), dans laquelle elle-même, se localise une minuscule phase parfaitement ronde (sulfure) (photo 3 h). Clozel (1997) et Colombel (1997) ont caractérisé également des vitrifiat de Refiom. Leurs résultats sont en accord avec ceux présentés ci-dessus.

\section{Localisation des éléments polluants}

Dans les échantillons " vitreux », les éléments potentiellement toxiques se distribuent de manière très contrastée entre les spinelles et le verre. Les premiers concentrent très fortement $\mathrm{Cr}$ et $\mathrm{Zn}$ et montrent une zonation chimique représentée par une bordure enrichie en $\mathrm{Al}$ et $\mathrm{Zn}$ et un cœur enrichi en $\mathrm{Cr}$.

Par rapport à la composition globale du matériau, le facteur de concentration dans les spinelles varie de 70 à 110 pour $\mathrm{Cr}$ et se situe aux alentours de 7-8 pour $\mathrm{Zn}$. Tout le $\mathrm{Pb}$ $(0,9 \%)$ et une grande quantité du $\mathrm{Zn}$ initial $(5,55 \%)$ restent dans la matrice vitreuse.
Dans les échantillons « cristallisés », les spinelles concentrent fortement $\mathrm{Cr}$ et $\mathrm{Zn}$. Ils sont zonés, étant plus riches en $\mathrm{Al}$ en bordure ( $41 \%$ poids d'oxyde contre $33 \%$ au centre) et plus riches en $\mathrm{Cr}$ au centre $(41 \%$ contre $31 \%$ en bordure). Le facteur d'enrichissement par rapport à la composition globale est de 9 pour $\mathrm{Zn}$ et varie de 40 à 50 pour $\mathrm{Cr}$. La grande particularité de ce type d'échantillon est de contenir des billes de phosphures, sulfures et alliages métalliques, très riches en métaux toxiques.

Les analyses des diverses phases des billes millimétriques sont données dans le tableau 2. Les phases majeures (e) et interstitielles $(f)$ contiennent de très fortes teneurs en $\mathrm{Cr}(22,16$ et $8,95 \% \mathrm{pd})$. Elles contiennent également quelques traces de $\mathrm{Pb}(0,32$ et $0,15 \% \mathrm{pd})$. C'est dans la phase arrondie $(\mathrm{g})$ que se concentrent $\mathrm{Sn}$ et $\mathrm{Sb}(8,85$ et 3,97 \% pd). La minuscule phase ronde (h) est un sulfure de $\mathrm{Cu}$ et Fe qui contient à plus faible teneur tous les métaux. Les petites phases opaques dispersées dans le verre sont en majorité des sulfures de $\mathrm{Cr}(45,15$ et 33,52 \% pd) (tableau 3, analyses 4 et $5)$, contenant du Fe $(3,68)$ ou Fe et $\mathrm{Zn}(5,87$ et 6,09$)$, et des sulfures de Fe contenant un peu de $\mathrm{Cr}(1,74)$ et $\mathrm{Cu}(1,76)$ (tableau 3, analyse 2 ) et des traces de $\mathrm{Pb}(0,23)$, ou encore des sulfures de $\mathrm{Fe}$ et $\mathrm{Cr}(19,37)$ contenant un peu de $\mathrm{Zn}$ $(3,66)$ (tableau 3 , analyse 3 ). Elles peuvent être également formées par du fer métallique (tableau 3, analyse I) dont les taux de carburation vont jusqu'à la fonte (visible en observation métallographique). Dans le dernier cas, c'est un phosphure de fer (tableau 3, analyse 6) contenant nombre de métaux toxiques tels que Co $(2,14 \% \mathrm{pd}), \mathrm{Ni}(5,55)$, Sn $(1,83)$ et $\mathrm{Sb}(1,60)$ et à plus faible teneur du As $(0,59), \mathrm{Cu}$ $(0,34), \mathrm{Zn}(0,14)$ et $\mathrm{Cr}(0,15)$. II n'a pas été fait mention de $\mathrm{Ba}$ car les teneurs se situent en dessous du seuil de détection de la sonde électronique. Néanmoins, il a souvent été observé que cet élément se concentrait dans la matrice vitreuse, ce qui semble être le cas ici pour les deux types de matériaux.

\begin{tabular}{|c|c|c|c|c|}
\hline \multicolumn{5}{|c|}{$\begin{array}{l}\text { Tableau } 2 \text { : Composition des phases } \\
\text { d'une grosse bille }\end{array}$} \\
\hline $\begin{array}{l}\% \text { pds } \\
\text { élmts }\end{array}$ & $\begin{array}{c}\text { majeure } \\
-\mathrm{e}-\end{array}$ & $\begin{array}{c}\text { interstitielle } \\
\text {-f- }\end{array}$ & $\begin{array}{l}\text { Ph. arrondie } \\
-g \text { - }\end{array}$ & $\begin{array}{l}\text { ph. ronde } \\
\text {-h- }\end{array}$ \\
\hline $\mathrm{Fe}$ & 53,15 & 75,16 & 4,47 & 18,73 \\
\hline$P$ & 22,77 & 14,87 & 0,44 & 0,87 \\
\hline $\mathrm{Cr}$ & 22,16 & 8,95 & 1,32 & 0,84 \\
\hline$S$ & 0,00 & 0,01 & 1,51 & 14,63 \\
\hline $\mathrm{Cu}$ & 1,27 & 0,78 & 78,98 & 61,99 \\
\hline $\mathrm{Zn}$ & 0,00 & 0,05 & 0.46 & 0,00 \\
\hline Sn & 0,00 & 0,00 & 8,86 & 0,84 \\
\hline Sb & 0,02 & 0,00 & 3,97 & 0,39 \\
\hline $\mathrm{Pb}$ & 0,32 & 0,15 & 0,00 & 0,00 \\
\hline Total & 99,69 & 99,97 & 100,01 & 98,29 \\
\hline
\end{tabular}


Tableau 3 : Composition des petites phases arrondies opaques ( $\ll-»$ en dessous du seuil de détection)

\begin{tabular}{lccllll}
\hline \% pds & & & & & \\
élmt & $-1-$ & $-2-$ & $-3-$ & $-4-$ & $-5-$ & $-6-$ \\
$\mathrm{S}$ & 1,89 & 36,85 & 37,10 & 40,15 & 40,97 & 0,28 \\
$\mathrm{Cr}$ & 0,16 & 1,74 & 19,37 & 45,19 & 33,52 & 0,15 \\
$\mathrm{Fe}$ & 91,85 & 56,81 & 37,57 & 3,68 & 5,87 & 67,82 \\
$\mathrm{Zn}$ & - & - & 3,66 & 0,00 & 6,09 & 0,14 \\
$\mathrm{Cu}$ & 0,60 & 1,76 & 0,46 & - & - & 0,34 \\
$\mathrm{Sb}$ & - & - & 0,11 & - & - & 1,60 \\
$\mathrm{~Pb}$ & 0,20 & 0,23 & - & - & - & - \\
$\mathrm{As}$ & - & - & - & - & - & 0,59 \\
$\mathrm{Sn}$ & - & - & - & - & - & 1.83 \\
$\mathrm{Co}$ & - & - & - & - & - & 2.14 \\
$\mathrm{Ni}$ & - & - & - & - & - & 5.55 \\
$\mathrm{P}$ & - & - & - & - & - & 12.94 \\
Total & $\mathbf{9 4 , 7 0}$ & $\mathbf{9 7 , 3 9}$ & $\mathbf{9 8 , 2 7}$ & $\mathbf{8 9 , 0 2}$ & $\mathbf{8 6 , 4 5}$ & $\mathbf{9 3 , 3 8}$
\end{tabular}

\section{INTERPRÉTATION}

\section{Conditions de cristallisation}

Dans les deux types de vitrifiats, les spinelles sont très riches en $\mathrm{Cr}$ avec une même zonation (plus riche en $\mathrm{Cr}$ au cœur et en $\mathrm{Al}$ et $\mathrm{Zn}$ en bordure). Le paramètre contrôlant ce phénomène est la température. Plus le spinelle cristallise à haute température, plus le $\mathrm{Cr}$ est incorporé en grande quantité. La grande différence entre les deux matériaux vient du Zn qui est incorporé en très grande quantité dans les spinelles du vitrifiat « vitreux » et de manière beaucoup moins importante dans ceux du vitrifiat "cristallisé ». Le paramètre contrôlant cette incorporation est tout simplement la teneur en $\mathrm{Zn}$ dans le bain fondu, qui est plus importante pour les échantillons vitreux. La forme arrondie des sulfures indique leur formation en phase liquide. Ils pourraient résulter de la démixtion d'un liquide riche en soufre du bain fondu restant après la formation des spinelles, des mélilites et des sphènes. En effet, au fur et à mesure de la cristallisation de ces trois types de cristaux, le liquide s'enrichit en S. Lorsque la teneur devient importante, et à cause des conditions réductrices du milieu, des petites gouttes s'individualisent en sulfures et alliages métalliques. Les phosphures sont formés par le passage de gaz réducteurs produits par l'oxydation des électrodes en graphite dans le bain fondu (billes à proximité des bulles) qui réduisent les éléments de ce bain et notamment le $P$, le $S$ et les métaux.

\section{Influence sur le taux de lixiviation}

Afin de savoir si la différence de minéralogie avait une conséquence sur le taux d'altération de ces matériaux, des tests simples de lixiviation ont été effectués. Ceci a permis de mettre en évidence un comportement complètement différent de ces deux vitrifiats. II faut rappeler que les échantillons " vitreux » présentent des teneurs en éléments toxiques beaucoup plus importantes que ceux des échantillons « cristallisés » sauf pour $\mathrm{Cr}$ et $\mathrm{Ba}$. La figure I présente la perte de masse normalisée des éléments polluants au cours de l'attaque, en fonction de la teneur du même élément dans le matériau sain. Le calcul de la perte normalisée se fait de la manière suivante :

$$
\mathrm{Nli}=(\mathrm{Ci} * \mathrm{~V}) /(\mathrm{Pi} * \mathrm{~S})
$$

$\mathrm{Nli}$ : perte de masse normalisée de l'élément i -en g. $\mathrm{m}^{2}$ -

$\mathrm{Ci}$ : concentration de l'élément $\mathrm{i}$ dans le lixiviat -en $\mathrm{g} . \mathrm{l}^{-1}$ -

$V$ : volume de la solution altérante

$S$ : surface d l'échantillon -en $\mathrm{m}^{2}$ -

$\mathrm{Pi}$ : pourcentage poids de l'élément i dans le verre sain -sans dimension-

Elle indique nettement que, comparativement à la teneur dans le matériau sain, le matériau essentiellement « vitreux »

Perte de
masse
normalisée
$-10^{-9} \mathrm{~g} / \mathrm{m}^{2}-$
dans la
solution
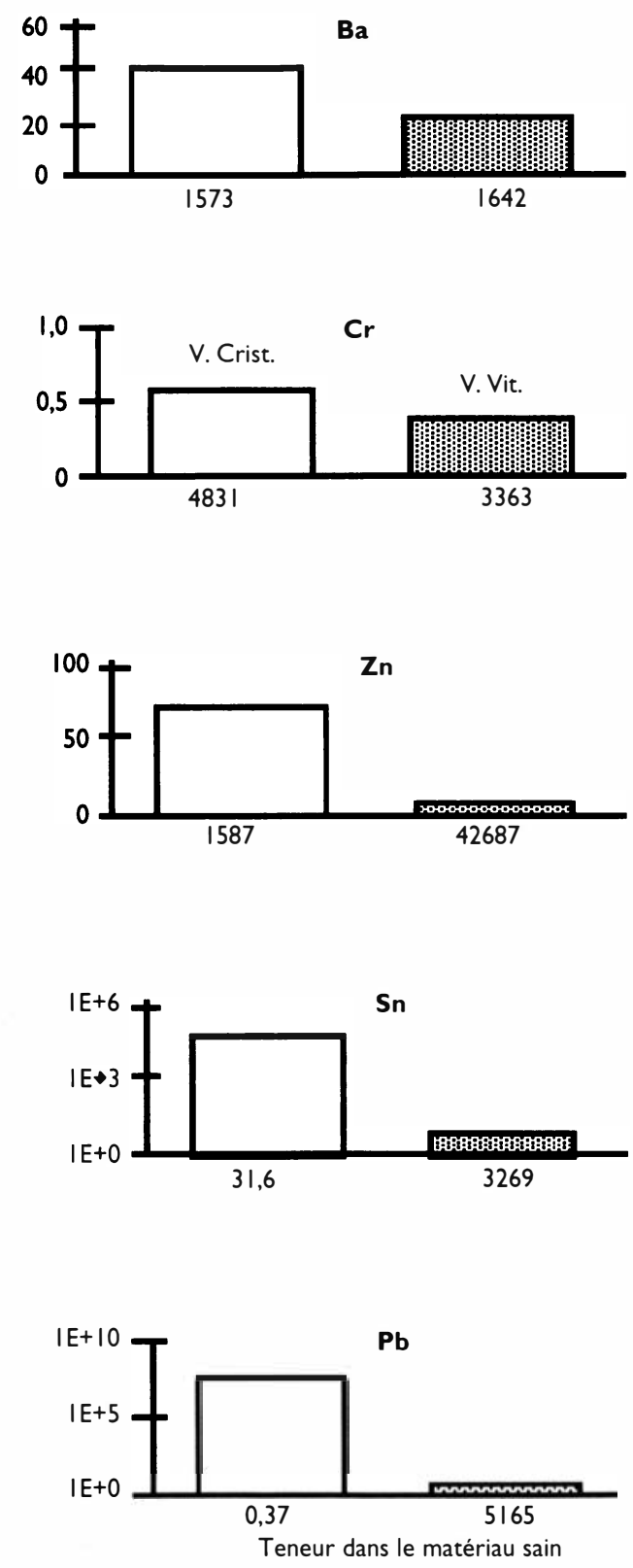

Figure I : Pertes de masse normalisées mesurées dans la solution après attaque des deux vitrifiats (cristallisé en blanc, vitreux en gris) pour quelques éléments polluants-pH $=\mathrm{I}$, solution $\mathrm{HCl}, 20^{\circ} \mathrm{C}$ 
relache beaucoup moins d'éléments toxiques que celui « cristallisé ». Le taux de perte des éléments est en relation directe avec leur localisation dans les différentes phases cristallisées et vitreuse. Ainsi, en ce qui concerne $\mathrm{Ba}$, les pertes sont sensiblement les mêmes pour les deux matériaux, car les teneurs dans les matériaux sont de même valeur et il se situe dans les deux cas dans la phase vitreuse attaquée. En ce qui concerne $\mathrm{Cr}$, la plus forte perte du matériau « cristallisé » est due principalement à sa localisation. Dans le vitrifiat " vitreux », $\mathrm{Cr}$ est localisé exclusivement dans les spinelles très résistants à l'altération ${ }^{[6]}$. Dans le matériau " cristallisé », il est également localisé dans les spinelles (avec un facteur d'enrichissement moins important) mais aussi dans les phosphures, sulfures et alliages métalliques, qui tous trois s'altèrent très rapidement.

Pour $\mathrm{Zn}, \mathrm{Cu}, \mathrm{Ni}, \mathrm{Co}, \mathrm{Pb}, \mathrm{Sn}$, As et $\mathrm{Sb}$, le phénomène est identique que pour $\mathrm{Cr}$ mais d'ampleur plus importante. Dans le vitrifiat " cristallisé », ces éléments se situent dans les billes de sulfures, phosphures et alliages métalliques (et également un peu dans les spinelles pour $\mathbf{Z n}$ ), alors que dans le vitrifiat vitreux, ces phases très altérables n'existent pas. $\mathrm{Pb}, \mathrm{Sn}, \mathrm{As}$ et $\mathrm{Sb}$ se localisent alors dans la matrice vitreuse. $Z n$ est incorporé dans le verre et dans les spinelles. La localisation de $\mathrm{Cu}, \mathrm{Ni}$ et $\mathrm{Co}$ dans ce vitrifiat amorphe n'a pas pu être déterminée avec précision du fait de leur faible teneur par rapport aux méthodes d'investigation, néanmoins ils pourraient se situer dans les cristaux de spinelle.

Cette localisation des éléments toxiques dans des phases réduites et peu stables (phosphures, sulfures et alliages) pour le vitrifiat cristallisé explique pourquoi ce matériau relache de plus grandes quantités de ces éléments alors que les teneurs dans le matériau sain sont moins importantes que pour le vitrifiat " vitreux ». C'est un problème de concentration des éléments dans des phases cristallisées qui s'altèrent rapidement.

\section{CONCLUSION}

La caractérisation chimique et minéralogique fine de deux types de vitrifiat de Refiom a été entreprise afin de localiser les éléments polluants dans les différentes phases formées et de comprendre la différence du taux d'altération entre les deux matériaux. Ce travail a montré que le matériau " vitreux " présente une minéralogie simple alors que celle du vitrifiat " cristallisé » est plus complexe et comprend des phases plus altérables concentrant les éléments polluants. Les tests de lixiviation ont corroboré ces observations montrant que, par rapport à la quantité d'éléments incorporés dans le matériau sain, le matériau « vitreux » relâche moins d'éléments polluants que celui " cristallisé », étant donc plus résistant à l'altération.

Un changement de composition chimique des produits de départ peut donc conduire à la fabrication de matériaux plus résistants, par un changement de la composition minéralogique. Certaines phases sont plus stables que d'autres et il est intéressant de favoriser leur croissance, sur l'exemple des spinelles. A l'inverse, il faut éviter la création de phases réduites (sulfures et phosphures) qui concentrent fortement les métaux toxiques et qui s'altèrent facilement.

Ce type de travail permet, connaissant la nature des phases formées, de cerner les conditions d'élaboration et de prévoir leur résistance avant même de mener des séries de tests de lixiviation qui peuvent s'avérer longs et coûteux. C'est un travail préliminaire qui est indispensable à la bonne connaissance des matériaux élaborés que l'on souhaite les plus résistants possible.

\section{Cécile Mahé-Le Carlier, Alain Ploquin, Jean-Jacques Royer}

Centre de recherches pétrographiques et géochimiques - CNRS Nancy BP 20 - 5450I Vandoeuvre-les-Nancy cedex

Remerciements

Cette étude a été financée en partie par EDF, le BRGM et par l'Ademe. Les auteurs remercient le Service commun d'analyses (Faculté des sciences, Vandoeurre-lès-Nancy) ainsi que le Service d'analyses des roches et minéraux (CRPG, Vandoeuvre-lès-Nancy) pour leur aide apportée à ce travail.

\section{Bibliographie}

[I] Meunier R., Le Boulch D., (1994) L'arc électrique pour le traitement de Refiom toxiques. Les Cahiers de l'Ingénierie, 52, 5-9.

[2] Meunier R., (1997) L'Arc électrique pour le traitement de Refiom toxiques, In : Procédés de solidification et de stabilisation des déchets, Nancy, 28 novembre- $\left.\right|^{\text {er }}$ décembre 1995, J.M. Cases, F. Thomas eds. 138-142, Société Alpine de Publications.

[3] Mahé-Le Carlier C. (1997) Caractérisation pétrographique et chimique d'analogues de déchets vitrifiés actuels : les scories de la métallurgie ancienne. Étude de l'altération naturelle et expérimentale. Thèse INPL Nancy, 337p.

[4] Colombel P. (1996), Étude du comportement à long terme de vitrifiats de Refiom. Thèse Poitiers 310p.

[5] Clozel B., Legendre O., (1997) Caractérisation minéralogique et cristallochimique de vitrifiats. In : Procédés de Solidification et de Stabilisation des Déchets, Nancy, 28 novembreler décembre 1995, J.M. Cases, F. Thomas eds., 53-57, Société Alpine de Publications.

[6] Arnold M., Bach Tuyet L., Blazy P., Febvay-Choffel L., Pineau J.L., Thauront J. (1997) Brevet International TrediTherm $n^{\circ} 27-122-14 P$. 